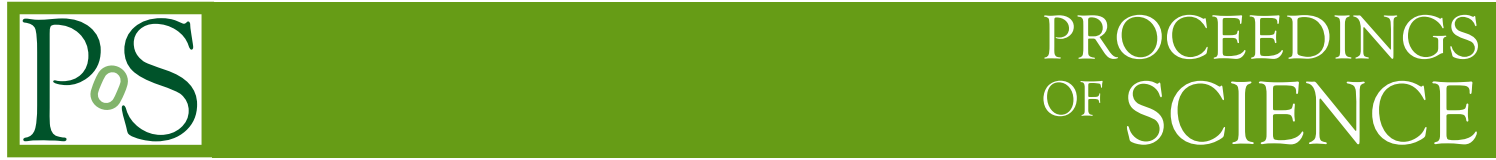

\title{
Saturday question and answer transcripts
}

\section{Thomas A. Trainor*}

CENPA 354290, University of Washington, Seattle, WA 98195 USA

E-mail: trainorehausdorf.npl.washington.edu

This is a transcript of the question-answer intervals following the Saturday talk presentations. In some cases notes were added during participant review of the transcript (sources are specified). Participants were: Rene Belwied, Charles Chiu, Tamas Csörgo Yuri Dokshitzer, Ricardo Rodriquez, Rainer Fries, Ahmed Hamed, Ulrich Heinz, Che-Ming Ko, Denes Molnar, Guy Moore, Lanny Ray, Thorsten Renk, Derek Teaney, Tom Trainor.

Workshop on Critical Examination of RHIC Paradigms - CERP2010

April 14-17, 2010

Austin Texas USA

${ }^{*}$ Secretary. 


\section{Charles Chiu}

Lanny: A word of caution about the STAR data that you showed. Please go back to where you show the yield vs $\phi_{s}$, where basically it falls off as you go from in plane to out of plane [p. 10]. Here's the question I've asked them: The problem is you've got this falling yield as a function of angle relative to the reaction plane. And, what they're claiming is that if you bin the data (experimentally you take a finite-width bin), and they take it on one side of the reaction plane and the other, and they're claiming to see this shift. I've asked them, there's this falling yield as you sweep across that bin, which is going to tend to make the side of the bin closer to the reaction plane look larger than the other. Just that alone will tend to make it look like it's skewed. Your later plots, where you say if they look at the distribution of yield relative to the trigger it tends to be skewed one way or the other, depending on which side of the reaction plane they're on. But that effect could also do that. I've asked them to remove that trivial effect and then see if there's anything left. And I'm quite sure that hasn't been done, so just a caution. It has nothing to do with your model, but to caution about the distribution you show later.

Charles: Of course, if we understand how you do the bin with the bias we will try to limit that also. Thank you.

Lanny: Yes, you can replicate [the effect].

Thorsten: The defining feature of the ridge is the long extent in $\eta$. On the first go in your model you sort of neglect that and say "we are only interested in every other feature." But I think you have a real problem, because your energy deposition, which is heating the medium, is very local in rapidity. And it happens rather late. It does not happen in the first half fermi or so. Suddenly, you need to get this local energy deposition stretched across two, three, four units in rapidity. How do you do that?

Charles: Either you think of that, or you think of the fact that at the very early time you have a very small volume (geometrically, everybody is close together). If you take into account the quantum mechanical uncertainties within the particular region, essentially everybody interacts with everybody else. In that particular domain some of the constituents naturally become leading particles ...

Thorsten: You're claiming that at $1 \mathrm{fm} / \mathrm{c}$ the whole medium is causally connected? That's when jet quenching starts being relevant. You don't get any energy deposition before because there's essentially no medium. The medium needs some formation time.

Charles: How about the following scenario? Within the $1 \mathrm{fm}$ there are interactions between potential leading particles and something which would be jets (trigger), everybody interacting with each other. Imagine the situation where when a leading particle starts to stretch out, it remembers that at this particular $1 \mathrm{fm}$, at the early time actually it touched base with the trigger particle. This is my question to you.

Thorsten: No, because it violates causality. By the time jet quenching starts you need first to form a medium, before you can quench anything. Without the medium there is no interaction with the medium. That takes some time because the medium is reasonably soft. If you translate that into a time scale it's difficult to get at very early times. Then your hard probe starts interacting with the medium. Then there's LPM interference. It takes some time to open up radiation phase space. It's very hard to get massive radiation initially. Typically, if you do calculations in hydro, jet quenching 
starts...the peak of energy deposition in jet quenching occurs in 3-5 fm. By that time you have no chance at all to get anything to forward rapidity.

Charles: OK, let me ask you the following question. Do you buy the flux-tube model?

Thorsten: I buy that the flux-tube model at least has a potential mechanism of getting largerapidity correlations, so that the strength of any initial state...

Charles: In that case, where does the large-rapidity correlation come from?

Thorsten: Because the flux tube is a large-rapidity correlation in the first place.

Charles: OK, here's my question to you. Suppose at the very early point you have something very close to rapidity zero. The other one is the leading particle. If, within the spacial domain these two things can talk to each other why couldn't we have a scenario where [correlations] are completely stretched out?

Thorsten: Because the flux tube is created by initial-state physics, and jet quenching is supposed to be final-state physics.

Charles: I was talking about initial state. In the CM system you have something which is coming out this way...

Thorsten: But that's not what you described in your talk. In your talk you described that an outgoing hard parton deposits energy into a medium.

Charles: I have not talked about the longitudinal part at all.

\section{Tamas Csörgo}

Tom: To be clear on this derivation, in your treatment of $R_{A A}$ you're assuming that $R_{A A}$ indicates an absorption of partons as opposed to an energy loss of partons and shifting of the [fragment] spectrum to the left [toward smaller $p_{t}$ ]. Is that correct?

Tamas: I don't assume that. Why do you think I assume it? I just said this is..., the only thing that I use is opacity, the optical constant of opacity. This means that opacity is the decrease of intensity ...[???] with distance. I don't derive it from underlying assumptions.

Tom: OK, thanks.

Tamas: When people study light, as in the example I showed, it is actually the diffraction of the light which makes this decrease. So, there are several possible mechanisms. It's just that intensity of the transmitted radiation is a little bit less than the incoming radiation. If the medium is not clean, but there are significant absorptions then you assume that his does not depend on the actual distance in the medium then you get this exponential law, exponential decrease.

\section{Guy Moore}

Mike: A comment on this jet energy loss calculation [infrared???] safe. When Rolf Baier first talked to me...right at the beginning, I guess '98, he said it was an $\alpha_{s}^{3}$ calculation, and the last time he did such a calculation, on $\Upsilon$ decay, it was off by a factor of ten. So, he was really concerned. Now you're saying "Forget that. You can't even use perturbation theory."

Guy: Yes. Certainly the part that has to do with describing the medium. If you ask about a very high energy jet and you model the medium and you just ask about the part where the jet is busy fragmenting then I think it's not hopeless. But, you have to be at a pretty high energy, because on 
the side of this deeply-falling spectrum relatively soft radiation is enough, and because the medium is relatively large, that soft radiation is pretty collinear, now your transverse-momentum scale is not very big.

Mike: So, why don't you say it's opaque? Big is not good enough.

Guy: Whatever we don't have we'll have soon.

Yuri: I can add, misusing my position as chairman, if we are going to speak about jet quenching then formally it is a calculable quantity, infrared safe, but you wash your hands there. But, if you start examining what is your real sensitivity to your ignorance of the infrared region it's enormous.

Ulrich: You came across as relatively pessimistic with regard to perturbation theory, and I don't think that should be the final impression, because what it shows is that even in the region where particles are weakly interacting on a two-by-two basis you can have very strong collective type of interactions, and everything that you have shown goes in the direction of making the idea of, in a collective sense, a strongly-coupled quark-gluon plasma more valid than I have seen before, and so...

Guy: Yeah, but I'm nervous about that interpretation.

Ulrich: What I take from this is that it gives us some hope that we are on the way towards understanding why hydrodynamics has worked for fifteen years much better than it should have. For the last ten years it has actually started working quantitatively.

Guy: There might be some truth to that, but I argued that the perturbation expansion should break down at $\alpha_{s}=0.1$ or even a little less. That's here, where the shear viscosity is 4 . And the issue is that part of your medium is becoming nonperturbative, when the part which is flying around and causing the shear viscosity still is perturbative. It's just that if the thing flying around wants to change its direction it has to interact with the part that is no longer under control. So, it's not quite as optimistic as you'd like to be. However, if you put in $\alpha_{s}=0.3$ then, contrary to what you claimed, a perturbative calculation tells you that $\eta / s$ should be "not so big", 0.6. And, there should be big corrections. And the first corrections you know about will make it lower. So, that's hopeful.

Ulrich: I'll change that line in my talk [laughter].

Ricardo: What you are saying is that entropy is a quantity that is not infrared safe.

Guy: Entropy is much more infrared safe than these dynamic quantities, viscosity, heavyquark diffusion. Entropy is safer. In fact it's known that at five times $T_{c}$ the entropy is not that far from the Stephan-Boltzmann [limit].

Ricardo: That was not actually my question. It was the following. In your calculations can you see, you're talking about infrared/collinear limit and things like that, at least when you work in vacuum in perturbative QCD those divergences are universal. You can factor them out. Do you see something like that in your calculations, that you have the universal type of divergence?

Guy: No.

Derek: If I can follow up on what Uli said and what Guy answered. You want to construct some kind of notion of quarks and gluons running around [Guy: Why?], that's what Uli wants, [Guy: In the medium, I'm not convinced you want that] and for hard particles you still have to have quarks and gluons there. And that has to propagate over a distance which is large compared to $1 / \pi T$. To think that you're going to squish into $1 / \pi T$ the small-angle scattering, the large-angle scattering, all of that into $1 / \pi T$, is kind of crazy. So, the point I would say is that the temperature at least has to be... there has to be some separation between the shear viscosity and the temperature, 
and once you put them at the same scale, it seems like very difficult to reconcile [in perturbation theory???].

Guy: Well, OK. So, I'm still hoping that this hard guy, if it has enough energy, it is like this one perturbative guy in this sea of goo. We should not try to describe the medium, the hot region out here, perturbatively. That's what I'm arguing.

Ulrich: Another comment where you can maybe destroy my optimism again. I though that I learned from your paper that what $\alpha$ should be is controlled by the structure of the collision term. And when the spectra are fixed we are in the hadronic phase because we are talking about hadrons. And we know the interactions between them phenomenologically, so we should be able to calculate what that $\alpha$ is. If I understand Derek correctly, for a hadronic scattering $\alpha$ should be close to 0 .

Guy: I'm not sure he said that. Let me emphasize this. This is the property of freezeout. Therefore, it should be a description of the system when the system is going to where the mean free path is comparable to the length of the system, or at least comparable to...you want that there is at most one more scattering to happen in the rest of the history of the system. I think that should happen when we are in a hadronic description. Some would argue that somehow that happens exactly as you are switching from partonic to hadronic descriptions. I think it should happen when you already have the hadronic description, because you're saying we have several fm mean free path. So, alpha should be determined by hadronic physics, but hadrons with $T \sim 160 \mathrm{MeV}$, momenta around $500 \mathrm{MeV} / \mathrm{c}$. Some of those cross sections are known. And that's right in the regime where inelasticity is becoming important. So, this $\alpha$ should be something that one should be able to model or determine from mostly-known hadronic physics.

Ulrich: These are not forward-peaked cross sections like pQCD and radiative cross sections, right? And that's why I thought $\alpha$ should be closer to 0 and the deviation would be...

Guy: $\alpha$ would be zero if those cross sections were all 2 by 2 and $1 / s$. But I didn't think that hadronic cross sections were well described by angular independence and $1 / \mathrm{s}$.

Derek: I don't agree with what you said, especially as you get out to $2 \mathrm{GeV}$. $\delta f$ is a complicated function of time, and just the same way that you have a $\tau \pi$ for the time dependence of $\pi$ you should have a $\tau \delta f$, which is a function of momentum. And, as you go out to this large momentum this $\tau \delta f$ becomes larger and larger. So I suspect that this region of momentum space is determined by earlier and earlier times. I really don't think it's determined by that last scattering close to freezeout.

Guy: So, do you think there's some good way of determining what this $\alpha$ should be?

Derek: No, I don't. But I do think that region of phase space of $v_{2}$ [????] around $2 \mathrm{GeV}$ was determined earlier than the bulk.

Guy: That's possible. The thing I want to emphasize that I definitely do believe is that this $\alpha$ here is a parameter of viscous hydro in describing these final-state spectra, whether you want it or not. And, what people have been doing is either not thinking about it or just assuming that it's zero. But that's not really fair. The value zero is not perturbative, not particularly theoretically motived.

Ulrich: My point was that you should be able to get a handle on it.

Guy: There may be ways to get a handle on it from hadronic physics. I would hope that there are. But it's certainly there, and you have to worry about it.

Derek: We argued in our paper Uli that in the hadron phase it should be closer to 1, because you have inelastic processes: you have a daughter come in and it splits into three other daughters, 
then it cascades like that. So, you lose all of your energy in a finite length. In that case we should get $\alpha=1$. So, we argued precisely the opposite of what you thought I said in our paper.

\section{Denes Molnar}

Yuri: Very clear final point. I wish all microscopic transportation practice was as reliable as your macroscopic transport theory.

Rene: When you compare to the BAMPS, to $2 \rightarrow 3$ [obscured], one thing that always bothers me, in the transport model how can you have a coupling constant as a free parameter? Why shouldn't there be a limit on the coupling constant, at which point transport is not valid anymore?

Denes: If you want validity you had the talk from Guy. When you apply this transport you really are applying it in a regime where you cannot prove its validity. But this is the only nonequilibrium tool you have that you can solve. If you want to get some feeling about non-equilibrium dynamics there is nothing else you can do. It's very similar to the hydro too, so when you buy into the hydro you don't know whether it was justified. Parametrically you know when it was justified, but quantitatively you don't. And you look at the data-it's kind of too late already. You should have known from theory whether you should have made this transition to the hydro...

Rene: I can look at Guy's plot and say clearly there is $\alpha_{s}$ of $0.6 \ldots$

Denes: To some extent I believe that the higher numbers here basically boil down to what Guy was showing, that even (this is basically higher order), but even this higher order doesn't capture everything. So, they have this fudge factor there to perhaps sum up more, but it's clearly a fudge factor. We don't know what is out there. This is the question Edward keeps asking: if the $2 \rightarrow 3$ matter how about $2 \rightarrow 4,3 \rightarrow 3$ and so on, and how can we sum up all those contributions. So, I don't advocate this particular choice but I understand where it's coming from, that you don't have a rigorous control over your perturbation expansion.

Rene: Maybe my question's more basic from an experimentalist's view. If I include multitransport processes [as above] does that at the same time allow me to raise the coupling constant, because I couple more? Is that the same thing?

Denes: This is basically a dial for them to have more rate. The way I look at this, this is their knob to have more rate.

Rene: But is the knob free when you do transport? There must be some connection between the coupling constant and the multitransport condition, right?

Denes: Transport is a truncation over ignoring certain processes. If you cannot tell on perturbative grounds that those processes are unimportant then you're in trouble. That's what Guy is finding, that if he takes another class of processes it should be parametrically less important. $\mathrm{He}$ finds that they are more important than the order that he computed. So, you lose that particular control already, and there is no way to smuggle it back. It's not going to come back because you run BAMPS. But, the way I look at [???] is that immediately a factor four is hiding here [if] I change this parameter. But if I can understand a lot of phenomena with a factor 2 adjustment in $\alpha_{s}$ then I'm happy with that model, if it can explain a lot of phenomena, even though I know it's not perturbative, I know I cannot derive this from first principles, but if this is just a simple readjustment of one parameter in the framework which can explain a lot of things then it's useful. It's a little bit like condensed matter when you have systems you cannot justify your approximations but you have a 
practical drive, that you want to understand something for a practical purpose, a practical aspect, with one parameter you can explain all these things then that's good. But I don't know what the answer is because I'm still working on trying to cross check this thing, which hopefully in a year will happen.

Rainer: In your hydro study did you look at $v_{2}$ also?

Denes: This is still in $0+1$, because I wanted to make sure that I had an easy access to the $\delta f$. The problem is once you do transverse expansion $\delta f$ also depends on where you are in the transverse space. But it's possible. It will be much more difficult to look into [....] $\delta f / f$, because you also integrate over space. It's hopeless to get information so [???] in phase space. It's possible, but it will be more difficult than this simple thinking.

Rainer: One more comment about the scaling you showed at the end, with the quarks coalescing into hadrons, one thing that experimental data tell us is that if you look at protons and deuteron they would be coalesced particles constituents, they see the scale.

Denes: Yes, and we don't question that. For both Che-Ming and I, the main difficulty is your deuteron is there and the most likely production is coalescence, and there's nothing else, no competition, on one hand. On the other hand is there's no issue with energy or momentum conservation. The binding is small. And third, there is no constraint that all your protons and neutrons must end up in a deuteron. Whereas here, in his calculation, you have to have a mechanism which does that, so he does it some way. Here, I'm only plotting coalescence contribution from my model. This is really the rare event coalescence, so just do integrals over some hypersurface. But the main difference here is the time: it's fuzzy. In fragmentation the picture would change, even worse, but I didn't want to show that.

Che-Ming: There's another reason, because for hadron the $\phi_{\Omega}$, the size is very small, so $\delta x$ is very small. The $\delta p$ is large. So, beside the spacial-momentum correlation large $\delta p$ the naïve coalescence...you do that with a simple scaling. But the deuteron is different, has a huge size with radius $2.5 \mathrm{fm}$. This is weakly bound. So, $\delta x$ is large and $\delta p$ is tiny. So, deuteron is closer to naïve coalescence, beside the space-momentum correlations. So, for low-momentum deuteron and low-momentum proton they follow the scaling very well.

Ahmed: No matter what you do with the fit parameters or the initial-state parameters in hydro it will not describe at the same time HBT, spectra and $v_{2}$. Is this statement correct?

Denes: Yes. If you do hydro and you don't do any kind of hadronic transport, you just do the Cooper-Frye and assume that the gas of particles that you get in the end is what you observe then it [your statement] is true. Unless you go to some very strange hydrodynamic initial conditions. Basically, for HBT you need somehow initial flow, so people have to make arguments that the system is already flowing radially outwards and you can try to get the radii. And for spectra I think that the difficulty is that the chemical composition doesn't come out right. So, even though the shape of elliptic flow and the shape of the protons [spectrum?] could be fine, because this plot here is actually somewhat achieved, because here the spectra of the protons are also off. This is a calculation where you stop at $120 \mathrm{MeV}$. By then the chemical composition was .... too few protons, so they only fit to the shape. But, the yield is totally off. And within hydro there is no way to patch it. People tried partial chemical equilibrium, all this kind of weird approximate ideas, but they don't quite work. So, only Derek with his RQMD black box managed to get close to data. Nobody tried to reproduce it. He doesn't want to touch it again. That's basic real life. 
Guy: I just wanted to clarify this point about this $\alpha$, the power behavior expected in the perturbative equilibrium. Our emphasis was that you should expect this to be from... what value you get will depend on what processes, what matrix elements you put in. And, our claim was that if you put in inelastic processes with cross section which doesn't fall as $1 / s$ of the pair scattered then you get it away from zero. If you only have $2 \rightarrow 2$ I think there are a lot of theories where it turns out to be close to zero.

Denes: You mean the graph parameter? Yes.

Guy: So, I'm not surprised that you find a value close to zero, because you're just doing $2 \rightarrow 2$ scattering.

Denes: I'm not surprised either. I'm not saying you guys were wrong. What I'm saying is that I want to know how accurate this is, not just some sort of linear response perturbative thing. I want to see whether by transport which goes some $20-30 \%$ away from equilibrium, how much is this computable?

Rene: Just a followup to what Ahmed said. If you look at the thermal freezeout surface only hadronically, if you look at a blast-wave fit you can have a parallel fit to the spectra, $v_{2}$ and HBT.

Denes: Except you never get this from hydro.

Rene: Then the point is the problem doesn't necessarily lie in the partonic hydro phase. It lies in the transport you do in the hadronic phase.

Denes: But you don't do... usually most people don't do anything in the hadronic transport. It's too much mess. Most theorists avoid this like plague. You guys could have a chance if you do it on your own.

Rene: All I'm trying to say is if you don't get it out of hydro the way you're running hydro right now it doesn't invalidate hydro for the partonic phase.

Denes: I don't see that. All I'm saying is that you cannot make any statement about whether hydro was OK against data unless you have something to say about all these four points. Because it's the full package that you test. You cannot test one ingredient. Because then you will be always uncertain about the others, and you won't know whether your ignorance over the other points is what gives you the result.

Che-Ming: I think you showed that for $\eta / s$ greater than 0.2 hydro is not valid, right? In the hadronic phase $\eta / s$ is rather large so hydro just stops around $T_{c}$ and then you have follow with hadronic evolution. Then all those problems can be resolved. The final state is the hadronic phase. You have to use RQMD or something. 